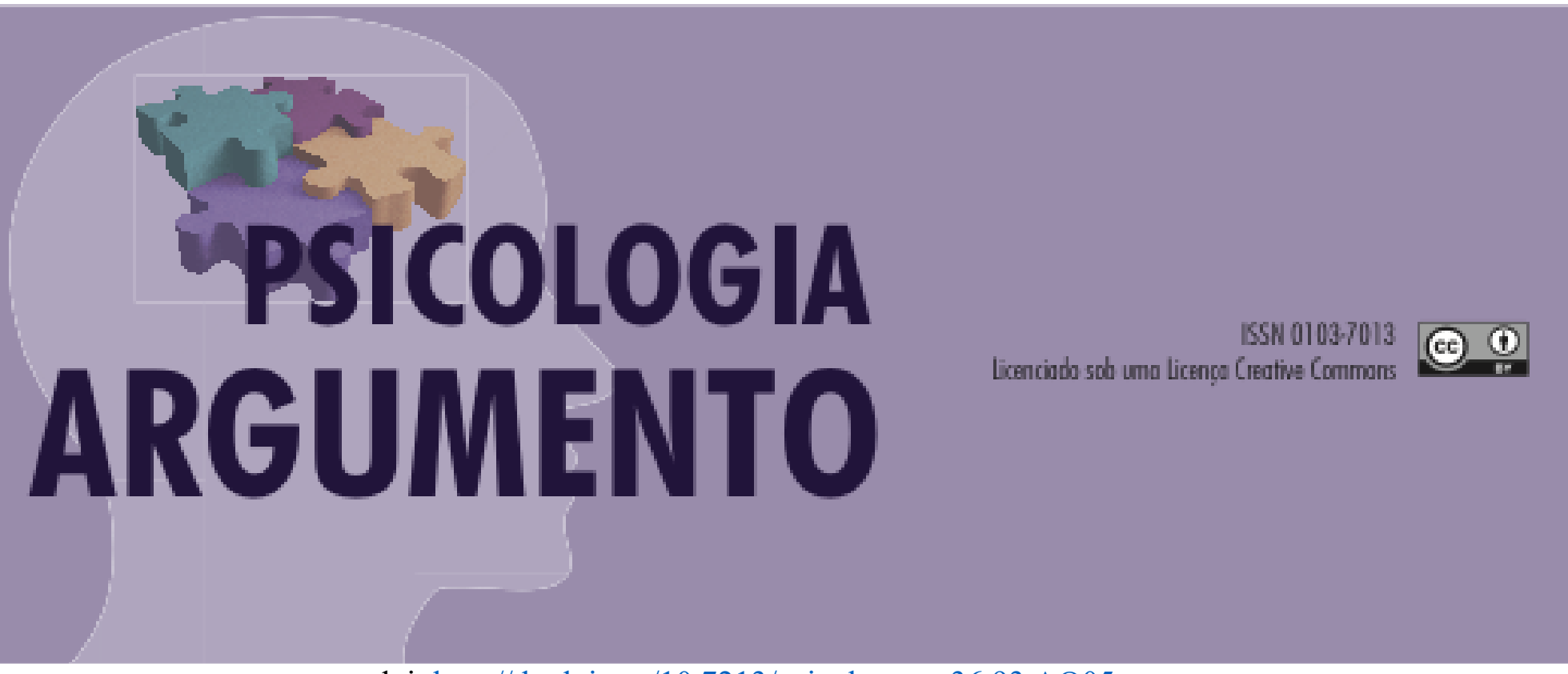

doi: http://dx.doi.org/10.7213/psicolargum.36.93.AO05

\title{
Melhora do desempenho de leitura com o uso de lâminas espectrais: revisão sistemática e meta-análise
}

Improvement in reading performance using spectral overlays: systematic review and meta-analyses

Mejora de la lectura con el uso de transparencias espectrales: revisión sistemática y meta-análisis

Douglas de Araújo Vilhena $^{[\mathrm{a}]}$, Márcia Reis Guimarães ${ }^{[\mathrm{b}]}$, Ricardo Queiroz Guimarães ${ }^{[\mathrm{c}]}$

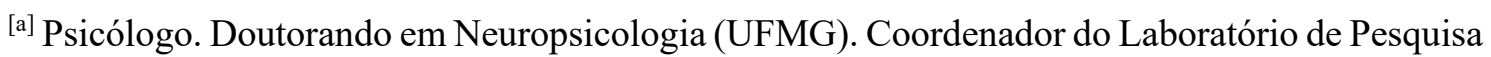
Aplicação à Neurociências da Visão (UFMG), Belo Horizonte, Minas Gerais, Brasil. E-mail: douglasvilhena@ufmg.br

${ }^{[b]}$ Médica. Doutorado em Oftalmologia (UFMG). Diretora do Departamento de Neurovisão do Hospital de Olhos de Minas Gerais. Brasil. E-mail: marciag2020@gmail.com

${ }^{\text {[c] }}$ Médico. Doutorado em Oftalmologia (UFMG). Diretor do LAPAN, do Hospital de Olhos de Minas Gerais e da Fundação Hospital de Olhos. E-mail: ricardoguimaraes@ufmg.br

\section{Resumo}

As lâminas espectrais são importantes ferramentas para minimizar distorções visuoperceptuais durante a leitura, aumentar o conforto visual e a fluência do leitor. Objetivo: Realizar uma revisão sistemática de todos os estudos que verificaram a prevalência da população beneficiada com o uso das lâminas espectrais. Método: Todos os títulos e resumos dos 225 artigos completos, de 1980 a 2017, foram avaliados independentemente por dois autores. Da seleção final, 26 estudos sobre a prevalência do uso de lâminas espectrais foram lidos na íntegra. Resultados: $63 \%$ da população em geral informam melhora perceptual da qualidade do texto com o uso das lâminas. A prevalências por meio do uso prolongado da lâmina, por pelo menos dois meses, revela um 
índice de $30 \%$ da população geral. Por fim, sob uso de lâminas, $33 \%$ da população melhorou a velocidade de leitura, quantificada via Rate of Reading Test, tendo $18 \%$ apresentado ganhos de moderados a robustos. Os grupos clínicos que apresentam maior probabilidade de ganhos na velocidade de leitura com o uso da lâmina espectral são, respectivamente: Síndrome de Tourette, Esclerose Múltipla, Transtorno do Espectro Autista, Dificuldade de Leitura. Conclusão: grande proporção da população apresenta ganhos na qualidade visual com o uso das lâminas espectrais, sendo esses índices maiores nos transtornos com hiperexcitabilidade cortical.

Palavras-chave: Síndrome de Irlen. Estresse visual. Leitura. Remediação da leitura. Intervenção precoce. Dificuldade de leitura.

\begin{abstract}
Spectral overlays are important interventions to reduce visual-perceptual distortions during reading, providing more comfort, fluency to the reader. Objective: systematically review all studies that assessed the population impact of the spectral overlays. Method: All titles and abstracts of 225 complete articles, from 1980 to 2017, were evaluated independently by two authors. In the final selection, 26 studies on the prevalence of the use of colored overlays were read in full. Results: $63 \%$ of children in the general population inform perceptual improvement in text clarity with the use of overlays. The prevalence with the sustained use of at least two months is revealed in $30 \%$ of the general population. Finally, under the use of overlays, $33 \%$ of the population improved reading speed, quantified via Rate of Reading Test, with $18 \%$ presenting moderate to robust gains. The clinical groups that are most likely to gain reading speed with the use of overlays are, respectively: Tourette Syndrome, Multiple Sclerosis, Autistic Spectrum Disorder, Reading Difficulty. Conclusion: a large proportion of the general population improves their visual quality with the use of spectral overlays, with disorders with cortical hyperexcitability presenting higher percentage.
\end{abstract}

Keywords: Irlen Syndrome. Visual Stress. Reading. Reading Remediation. Early Intervention. Reading difficulty.

\title{
Resumen
}

Las transparencias espectrales (overlays) son intervenciones importantes para reducir las distorsiones viso-perceptivas durante la lectura, proporcionando más comodidad y fluidez al lector. Objetivo: revisar sistemáticamente todos los estudios que evaluaron el impacto de los overlays en la población. Método: Todos los títulos y resúmenes de 225 artículos completos, de 1980 a 2017, fueron evaluados de forma independiente por dos autores. En la selección final, 26 estudios sobre la prevalencia del uso de los overlays fueron leídos en su totalidad. Resultados: $63 \%$ de los niños de la población general informan una mejoría perceptual en la claridad del texto con el uso de los overlays. La prevalencia con el uso contínuo de al menos dos meses se revela en el 30\% de la población en general. Finalmente, con el uso de los overlays, 33\% de la población mejoró la velocidad de lectura, cuantificada a través de la Prueba de Tasa de Lectura, con 18\% presentando mejorías de moderadas a robustas. Los grupos clínicos que tienen más probabilidades de mejorar la velocidad de lectura con el uso de los overlays son, respectivamente: Síndrome de Tourette, Esclerosis Múltiple, Trastorno del Espectro Autista, Dificultad de Lectura. Conclusión: una significativa proporción de la población presenta índices de mejoría en su calidad visual con el uso de las transparecias espectrales, siendo éstos mayores en individuos con transtornos relacionados a la hiperexcitabilidad cortical.

Palabras clave: Síndrome de Irlen. Estrés visual. Lectura. Remediación de Lectura. Intervención rápida. Dificultad de lectura. 


\section{Introdução}

Alguns leitores apresentam distorções visuoperceptuais durante a leitura de textos, como a presença de sombras e halos ao redor da palavra, espaçamentos irregulares em meio ao texto e percepção de movimento, como letras saindo do papel ou vibrando (Irlen \& Lass, 1989; Stein \& Walsh, 1997; Wilkins, Lewis, Smith, Rowland, \& Tweedie, 2001). A compensação física e cognitiva empregada para manter a leitura nessas situações gera, frequentemente, ardência ocular, lacrimejamento, cefaleia e fotofobia progressiva (Evans et al., 1996; Kriss \& Evans, 2005; Scott et al., 2002).

A sensação de instabilidade no texto ocorre inclusive em leitores com boa acuidade visual e na ausência de problemas refracionais e ortópticos (Monger, Wilkins, \& Allen, 2015; Scott et al., 2002). A dificuldade em visualizar o texto pode se apresentar em diferentes intensidades, provocando rápido cansaço visual ou prejudicando a leitura apenas após prolongado tempo de exposição, inclusive em leitores proficientes. A acentuada presença desses sinais e sintomas de dificuldades visuoperceptuais é referida na literatura como Síndrome de Sensibilidade Escotópica, Síndrome de Irlen, Síndrome de Meares-Irlen, Estresse Visual e Distúrbio do Processamento Visual.

As lâminas espectrais (spectral/coloured overlays) são folhas em acetato transparente em tonalidades específicas usadas sobrepostas no texto impresso ou na tela do computador. As lâminas tem se mostrado eficientes para minimizar as distorções visuoperceptuais e, consequentemente, melhorar o conforto durante a leitura, a deixando mais fluente e duradoura (Hlengwa, Moonsamy, Ngwane, Nirghin, \& Singh, 2017; Jeanes et al., 1997; Tyrrell, Holland, Dennis, \& Wilkins, 1995; Wilkins, Jeanes, Pumfrey, \& Laskier, 1996; Wilkins \& Lewis, 1999; Wilkins et al., 2001). Ao minimizar as distorções na leitura, com ganhos no conforto e fluência, é esperado uma aderência prolongada ao uso desse recurso em condições de sala de aula (Jeanes et al., 1997; Scott et al., 2002; Wilkins et al., 2001).

Uma vez que a luz branca é composta por diferentes comprimentos e frequências eletromagnéticas, as lâminas absorvem e refletem específicas faixas de luz do espectro visível. O bloqueio de certas faixas ativará de forma distinta as células fotorreceptoras na retina, alterando a transmissão da informação entre os sistemas visuais magno e parvocelular. O sistema magnocelular é considerado o caminho visual dominante na percepção do texto, pois media a capacidade de identificar rapidamente as letras e as suas 
posições na palavra, além de controlar a orientação visual da atenção, das fixações e sincronizações oculares (Chase, Ashourzadeh, Kelly, Monfette, \& Kinsey, 2003). O seu reequilíbrio com o sistema Parvocelular ajuda a redistribuir a informação visual nos neurônios corticais da área visual primária occipital, evitando áreas de hiperexcitação (Allen, Gilchrist, \& Hollis, 2008; Wilkins et al., 2001). Assim, a específica tonalidade da lâmina (ex., amarelada, azulada) pode aumentar ou diminuir a transmissão do sistema magnocelular, reequilibrando o processamento neurovisual (Croyle, 1998; Solan, Ficarra, Brannan, \& Rucker, 1998).

Desde a década de 1980 a melhora da leitura com o uso da lâmina espectral tem sido documentada, com diferentes populações, parâmetros e resultados. Na nossa revisão nos propomos a rever os seguintes aspectos. Qual a porcentagem média de leitores que apresenta melhora da qualidade visual do texto com o uso da lâmina espectral? Existe diferença entre os resultados de amostras populacionais em comparação a sujeitos com dificuldade de leitura e outros grupos clínicos? Quantos leitores manteriam o uso prolongado da lâmina selecionada durante o seu processo de aprendizado? Quantos leitores apresentam melhora quantitativa na habilidade de leitura com as lâminas?

Para responder essas perguntas, foi realizada uma revisão sistemática da literatura com meta-análise para verificar quantos leitores da população em geral e de grupos clínicos se beneficiariam com o uso de lâminas espectrais. Foi quantificada a prevalência via a escolha por meio do autorrelato, via o seu uso prolongado por pelo menos dois meses, e também pelo ganho na velocidade de leitura.

\section{Método}

\section{Revisão Sistemática}

Bancos de dados foram pesquisados por meio do Portal de Periódicos da Coordenação de Aperfeiçoamento de Pessoal de Nível Superior (CAPES) no sistema da Universidade Federal de Minas Gerais. Artigos experimentais completos em português ou inglês, de 1980 a 2017, foram pesquisados com as seguintes expressões no Título, Resumo ou Palavras-chave: lâminas coloridas/espectrais (Coloured/Spectral Overlays), Irlen, Meares-Irlen, Síndrome de Sensibilidade Escotópica (Scotopic Sensitivity Syndrome), Estresse Visual (Visual Stress). Banco de dados consultados: ERIC, PubMed, 
ScienceDirect, Scopus. Os seguintes bancos de dados não retornaram resultado: LILACS, PePSIC, SciELO.

Todos os títulos e resumos dos 225 artigos foram avaliados independentemente por dois autores. Foram inclusos retrospectivamente no banco de dados duas dissertação de mestrado (Garcia, 2016; Leão, 2018) e uma monografia de graduação Universidad de Cuenca (Bernal, 2011). Da seleção final, 26 estudos experimentais ou observacionais sobre o uso de lâminas espectrais foram selecionados e lidos na íntegra para compor a presente revisão sistemática.

\section{Instrumento}

O Rate of Reading Test (RRT) é o teste de leitura mais utilizado nas publicações científicas sobre as lâminas. O teste mede a velocidade de leitura em voz alta, sendo composto por 15 palavras curtas de alta frequência, repetidas e organizadas aleatoriamente em cada uma das 10 linhas (150 palavras no total), com fonte Times New Roman 9pt e espaçamento simples (Wilkins et al., 1996). Esse formato do RRT, construído com base na teoria da hiperexcitabilidade cortical, acentua os sintomas de estresse visual, permitindo analisar em menor tempo o efeito da lâmina na leitura (Wilkins, 1995; Wilkins et al., 2001). O RRT passou por diferentes validações, sendo que o desempenho nesse instrumento não pode ser explicado por problemas refrativos, ortópticos ou pelos efeitos placebo (Bouldoukian, Wilkins, \& Evans, 2002; Monger et al., 2015; Wilkins \& Lewis, 1999). Para eliminar o efeito de treino, a aplicação do RRT é composta por uma fase de treino para a habituação, seguida por quatro leituras intercaladas com e sem a lâmina espectral ideal (procedimento ABBA).

\section{Análise Estatística}

A diferença significativa entre dados categóricos/nominais foi determinada pelo Teste de Chi-Quadrado $\left(\chi^{2}\right)$ e com significância $(p)$ estipulada em 0,05. 


\section{Resultados}

As lâminas mais frequentemente utilizadas nas publicações científicas são as Irlen Overlays, que foram desenvolvidas na década de 1980 pela psicóloga educacional Helen Irlen, e as Intuitive Overlays, desenvolvidas pelo Prof. Emérito Arnold Wilkins (1994). Esses dois tipos de lâminas espectrais são compostos por 10 tonalidades, que podem ser combinadas para formar outras cromaticidades. A seguir, serão descritos diferentes parâmetros para a identificação da proporção de pessoas que fazem o uso das lâminas espectrais, tanto na população geral quanto clínica. Para a análise dessa prevalência, considerou-se o autorrelato (critério qualitativo), o uso prolongado voluntário (validade prática), e a melhora no desempenho de leitura (critério quantitativo).

\section{Prevalência por meio do autorrelato}

De acordo com a Tabela 1, de 40 a $88 \%$ das crianças da população geral relataram melhora no conforto visual e na nitidez do texto com o uso das lâminas espectrais (Jeanes et al., 1997; Scott et al., 2002; Tyrrell et al., 1995; Wilkins et al., 1996; Wilkins \& Lewis, 1999; Wilkins et al., 2001). A união das onze amostras de crianças e adolescentes, descritas na Tabela 1, totaliza 1756 alunos, dos quais 1108 (63\%) escolheram pelo menos uma lâmina espectral. Porcentagens maiores foram encontradas nos estudos de Miller (1985) e Evans e Joseph (2002), onde 88\% e 89\% dos universitários, respectivamente, relatam melhora qualitativa da leitura com as lâminas. Comparando esses dois grupos etários, a melhora visual com o uso da lâmina espectral foi maior em adultos do que em crianças $\left(\chi^{2}=47,01, p<0,0001\right)$.

Considerando grupos clínicos, a melhora visual foi maior em crianças com dificuldade de leitura do que os jovens da população em geral $\left(\chi^{2}=57,24, p<0,0001\right)$. Em crianças com dificuldade de leitura, o índice de melhora perceptual do texto com a lâmina varia de 71 a 100\% (média de $87 \%$, amostra total $=268$, autorrelato $=232$ ) (Bernal, 2011; Kriss \& Evans, 2005; Northway, 2003; Tyrrell et al., 1995). 
Tabela 1.

Prevalência, em diferentes populações, do autorrelato de melhora na qualidade da leitura com as lâminas espectrais

\begin{tabular}{|c|c|c|c|c|}
\hline Pop & Amostra & $\mathbf{n} / \mathbf{N}$ & $\%$ & Estudo \\
\hline \multirow{12}{*}{ 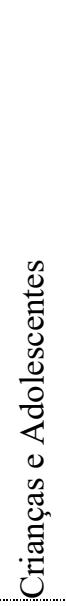 } & Grupo controle, $11-18$ anos & $26 / 39$ & $67 \%$ & Hollingsworth (2015) \\
\hline & Seleção aleatória, 5-11 anos & $47 / 93$ & $51 \%$ & Jeanes et al. (1997) \\
\hline & Seleção aleatória, $11-12$ anos & $32 / 59$ & $54 \%$ & Jeanes et al. (1997) \\
\hline & Seleção aleatória, 7-11 anos & $81 / 199$ & $41 \%$ & Scott et al. (2002) \\
\hline & Seleção aleatória, 10-12 anos & $132 / 153$ & $86 \%$ & Scott et al. (2002) \\
\hline & $\begin{array}{l}\text { Seleção não especificada, exclusão de } \\
\text { crianças com idade de leitura com mais de } \\
\text { dois anos abaixo da idade cronológica, } 8-16 \\
\text { anos }\end{array}$ & $23 / 40$ & $58 \%$ & Tyrrell et al. (1995) \\
\hline & Seleção aleatória, 8-11 anos & $38 / 77$ & $49 \%$ & Wilkins et al. (1996) \\
\hline & Seleção aleatória, 7-11 anos & $81 / 203$ & $40 \%$ & Wilkins e Lewis (1999) \\
\hline & Seleção aleatória, 8-10 anos & 78/89 & $88 \%$ & Wilkins et al. (2001) \\
\hline & Seleção aleatória, 8-12 anos & $314 / 378$ & $83 \%$ & Wilkins et al. (2001) \\
\hline & Seleção aleatória, 6-8 anos & $256 / 426$ & $60 \%$ & Wilkins et al. (2001) \\
\hline & TOTAL & $1108 / 1756$ & $63 \%$ & \\
\hline \multirow{3}{*}{$\frac{*}{\stackrel{*}{0}}$} & $\begin{array}{l}\text { Seleção espontânea, amostra } \\
\text { representativa, } 18-44 \text { anos }\end{array}$ & $101 / 113$ & $89 \%$ & Evans e Joseph (2002) \\
\hline & $\begin{array}{l}\text { Seleção amostral não especificada, } \\
\text { universitários com e sem dificuldade leitora }\end{array}$ & $57 / 65$ & $88 \%$ & Miller (1985) \\
\hline & 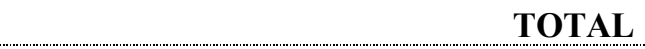 & $158 / 178$ & $89 \% *$ & \\
\hline \multirow{8}{*}{ 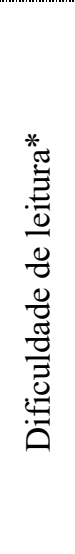 } & Amostra de conveniência, $7-12$ anos & $17 / 24$ & $71 \%$ & Bernal (2011) \\
\hline & $\begin{array}{l}\text { Amostra de conveniência, disléxicos, 7-12 } \\
\text { anos }\end{array}$ & $27 / 32$ & $84 \%$ & Kriss e Evans (2005) \\
\hline & Amostra populacional, 7-10 anos & $58 / 58$ & $100 \%$ & Leão (2018) \\
\hline & $\begin{array}{l}\text { Amostra de conveniência, disléxicos, 6-12 } \\
\text { anos }\end{array}$ & $50 / 64$ & $78 \%$ & Northway (2003) \\
\hline & $\begin{array}{l}\text { Idade de leitura de um a dois anos abaixo da } \\
\text { idade cronológica, } 8-16 \text { anos }\end{array}$ & $9 / 12$ & $75 \%$ & Tyrrell et al. (1995) \\
\hline & $\begin{array}{l}\text { Idade de leitura de três a cinco anos abaixo } \\
\text { da idade cronológica, } 14-16 \text { anos }\end{array}$ & $6 / 6$ & $100 \%$ & Tyrrell et al. (1995) \\
\hline & $\begin{array}{l}\text { Amostra estratificada, presidiários com } \\
\text { desconforto e dificuldade de leitura de } \\
\text { moderada a severa, 16-67 anos }\end{array}$ & $65 / 72$ & $90 \%$ & Whichard et al. (2000) \\
\hline & TOTAL & $232 / 268$ & $87 \% *$ & \\
\hline
\end{tabular}

*Chi-Quadrado com diferença significativa em relação ao grupo de Crianças e Adolescentes, $\mathrm{p}<0,05$.

\section{Prevalência por meio do uso prolongado}

Diferentes estudos longitudinais demonstraram a prevalência do uso prolongado das lâminas espectrais nas atividades diárias de leitura, com no mínimo dois meses de intervalo após a seleção da lâmina por meio do autorrelato (ver Tabela 2). Em crianças, o uso prolongado das lâminas espectrais por pelo menos dois meses após a sua prescrição ocorreu em 19\% da amostra (Wilkins et al., 1996), por pelo menos 3-4 meses em 1943\% (Jeanes et al., 1997; Scott et al., 2002), e 8-10 meses em 24-32\% (Jeanes et al., 1997; Wilkins \& Lewis, 1999; Wilkins et al., 2001). Em crianças com transtorno de aprendizagem da leitura, o índice do uso prolongado por 3 meses subiu para $63 \%(40 / 64)$ 
(Northway, 2003). Em suma, 299 das 1014 crianças (29,5\%) mantiveram o uso prolongado das lâminas por pelo menos dois meses.

Tabela 2.

Prevalência do uso prolongado das lâminas espectrais em crianças da população geral

\begin{tabular}{lccl}
\hline \multicolumn{1}{c}{ Tempo de uso } & Amostra (n/N) & Prevalência & \multicolumn{1}{c}{ Estudo } \\
\hline 2 meses & $15 / 77$ & $19 \%$ & Wilkins et al. (1996) \\
3 meses & $66 / 152$ & $43 \%$ & Jeanes et al. (1997) \\
4 meses & $31 / 94$ & $33 \%$ & Scott et al. (2002) \\
4 meses & $38 / 199$ & $19 \%$ & Scott et al. (2002) \\
8 meses & $135 / 426$ & $32 \%$ & Wilkins et al. (2001) \\
10 meses & $11 / 34$ & $32 \%$ & Jeanes et al. (1997) \\
10 meses & $3 / 32$ & $9 \%$ & Jeanes et al. (1997) \\
\hline
\end{tabular}

\section{Prevalência por meio do ganho no desempenho de leitura}

O desempenho de leitura é um importante parâmetro para verificar a intensidade dos benefícios das lâminas espectrais e a probabilidade do seu uso prolongado. As Tabelas 3 e 4 expõem os dados de todos os estudos que utilizaram o RRT para avaliar a influência das lâminas espectrais na velocidade de leitura. Em amostras populacionais, ao utilizar o critério de corte em $5 \%$, que é o habitualmente empregado como parâmetro inicial de melhora na velocidade de leitura, foi encontrada a prevalência de 19-36\% em crianças (Garcia, Momensohn-Santos, \& Vilhena, 2017; Kriss \& Evans, 2005; Singleton \& Henderson, 2007a; Wilkins et al., 1996; Wilkins et al., 2001), de 43\% em adolescentes (Singleton \& Henderson, 2007a), e de 24-38\% em universitários (Evans \& Joseph, 2002; Nichols, McLeod, Holder, \& McLeod, 2009). Não houve diferença estatística na prevalência entre esses três grupos etários $\left(\chi^{2}=0,02, p=0,89\right)$.

Em crianças a adultos com dificuldade de leitura, foram obtidos ganhos de pelo menos 5\% na velocidade de leitura em $41-57 \%$ da amostra, sendo esta prevalência estatisticamente maior do que a da amostra populacional $\left(\chi^{2}=8,53, p=0,0035\right)$. Da mesma forma, outros grupos clínicos também apresentam maior probabilidade de ganhos de pelo menos $5 \%$ no RRT, chegando a $92 \%\left(\chi^{2}=17,71, p=0,0001\right)$ em crianças com Síndrome de Tourette, a $77 \%\left(\chi^{2}=20,83, p=0,0001\right)$ em pacientes com esclerose múltipla, e a $74 \%\left(\chi^{2}=58,79, p=0,0001\right)$ em pacientes com Transtorno do Espectro Autista.

Ao utilizar um critério de corte moderado de $10 \%$ no RRT, foi verificada uma incidência de 12-14\% em crianças (Kriss \& Evans, 2005; Singleton \& Henderson, 
2007a), e de 22\% em adolescentes (Singleton \& Henderson, 2007a). Com o critério de corte mais rigoroso de melhora de $25 \%$ na leitura, foi encontrada uma prevalência de $5 \%$ em crianças de 6 a 8 anos (Wilkins et al., 2001) e de 2\% em adultos (Evans \& Joseph, 2002). Em amostras clínicas, esta proporção aumentou para $30 \%$ em pacientes com Transtorno do Espectro Autista (Ludlow, Wilkins, \& Heaton, 2006; A. K. Ludlow, A. J. Wilkins, \& P. Heaton, 2008; Whitaker, Jones, Wilkins, \& Roberson, 2016), e 31\% em pacientes com esclerose múltipla (Newman Wright, Wilkins, \& Zoukos, 2007). Em suma, $33 \%$ da população geral apresenta no mínimo ganhos leves na velocidade de leitura com o uso da lâmina espectral ideal, com cerca de 18\% demonstrando ganhos moderados. 
Tabela 3.

Meta-análise da prevalência (número de sujeitos/amostral total) que obtêm ganhos na velocidade de leitura no RRT com o uso da lâmina espectral ideal

\begin{tabular}{|c|c|c|c|c|c|c|c|}
\hline \multirow{3}{*}{ População } & \multirow{3}{*}{$\mathbf{N}$} & \multicolumn{5}{|c|}{ Melhora na velocidade de leitura no teste } & \multirow{3}{*}{ Referência } \\
\hline & & & & RRT & & & \\
\hline & & $>\mathbf{5 \%}$ & $>8 \%$ & $>10 \%$ & $>15 \%$ & $>25 \%$ & \\
\hline \multirow{8}{*}{ 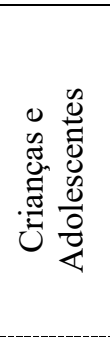 } & 68 & 21 & 15 & 12 & 9 & 2 & Garcia et al. (2017) \\
\hline & 32 & 8 & 7 & 4 & - & 1 & Kriss e Evans (2005) \\
\hline & 50 & 15 & 9 & 7 & - & - & Singleton e Henderson \\
\hline & 67 & 29 & 19 & 15 & - & - & $(2007 a)$ \\
\hline & 77 & 15 & - & - & - & - & Wilkins et al. (1996) \\
\hline & 426 & 153 & - & - & - & 21 & Wilkins et al. (2001) \\
\hline & $\mathrm{n} / \mathrm{N}$ & $241 / 720$ & $50 / 217$ & $38 / 217$ & $9 / 68$ & $24 / 526$ & \\
\hline & $\%$ & $33 \%$ & $23 \%$ & $18 \%$ & $13 \%$ & $5 \%$ & \\
\hline \multirow{8}{*}{$\frac{\stackrel{2}{0}}{\frac{E}{3}}$} & 113 & 38 & - & - & - & 2 & Evans e Joseph (2002) \\
\hline & 26 & 15 & 12 & 9 & - & - & Henderson et al. (2013) \\
\hline & 73 & 26 & - & 10 & - & - & Monger et al. (2015) \\
\hline & 74 & 18 & - & - & - & - & Nichols et al. (2009) \\
\hline & $\mathrm{n} / \mathrm{N}$ & $97 / 286$ & $12 / 26$ & $19 / 100$ & & $2 / 113$ & \\
\hline & $\%$ & $34 \%$ & $46 \% *$ & $19 \%$ & & $2 \% *$ & \\
\hline & $\chi^{2}$ & 0,02 & 6.53 & 0,01 & & 6.32 & \\
\hline & $\mathrm{p}$ & 0,89 & 0,011 & 0,75 & & 0,012 & \\
\hline \multirow{8}{*}{ 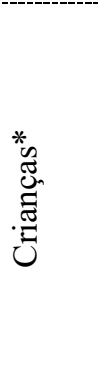 } & 58 & 27 & 22 & 17 & 10 & 8 & Leão (2018) \\
\hline & 32 & 15 & 11 & 10 & - & 1 & Kriss e Evans (2005) \\
\hline & 60 & 19 & - & - & - & - & Ritchie et al. $(2011)^{\Delta}$ \\
\hline & 22 & 9 & - & - & - & - & Singleton e Henderson \\
\hline & $\mathrm{n} / \mathrm{N}$ & $70 / 172$ & $32 / 90$ & $27 / 90$ & $10 / 58$ & $9 / 90$ & \\
\hline & $\%$ & $41 \%$ & $36 \% *$ & $30 \% *$ & $17 \%$ & $10 \% *$ & \\
\hline & $\chi^{2}$ & 3,19 & 5,09 & 5,94 & 0,39 & 6.32 & \\
\hline & p & 0,07 & 0,022 & 0,015 & 0,53 & 0,012 & \\
\hline \multirow{6}{*}{$\begin{array}{l}\text { * } \\
\stackrel{0}{0} \\
\frac{0}{3} \\
\frac{8}{4}\end{array}$} & 16 & 12 & 10 & 7 & - & - & Henderson et al. (2013) \\
\hline & 30 & 16 & - & - & - & - & Nichols et al. (2009) \\
\hline & $\mathrm{n} / \mathrm{N}$ & $26 / 46$ & $10 / 16$ & $7 / 16$ & & & \\
\hline & $\%$ & $57 \% *$ & $63 \% *$ & $44 \% *$ & & & \\
\hline & $\chi^{2}$ & 10,12 & 10,12 & 12,13 & & & \\
\hline & $\mathrm{p}$ & 0,0015 & 0,0015 & 0,0005 & & & \\
\hline \multirow{9}{*}{ 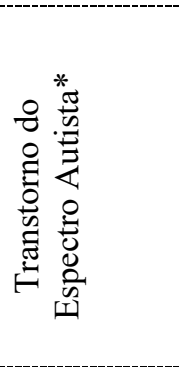 } & 19 & 15 & - & - & - & 6 & Ludlow et al. (2006) \\
\hline & 34 & 25 & - & - & - & 13 & Ludlow et al. (2008) \\
\hline & 15 & 12 & - & - & - & - & Ludlow et al. (2012) \\
\hline & 12 & 8 & - & - & 4 & - & Ludlow e Wilkins (2016) \\
\hline & 16 & 11 & 9 & 8 & 3 & 2 & Whitaker et al. (2016) \\
\hline & $\mathrm{n} / \mathrm{N}$ & $71 / 96$ & $9 / 16$ & $8 / 16$ & $7 / 28$ & $21 / 69$ & \\
\hline & $\%$ & $74 \% *$ & $56 \% *$ & $50 \% *$ & $25 \%$ & $30 \% *$ & \\
\hline & $\chi^{2}$ & 58,79 & 8,69 & 9,93 & 1,89 & 58.40 & \\
\hline & $\mathrm{p}$ & 0,0001 & 0,003 & 0,0016 & 0,17 & 0,0001 & \\
\hline \multirow{4}{*}{ 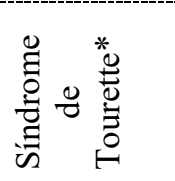 } & $\mathrm{n} / \mathrm{N}$ & $11 / 12$ & - & - & $6 / 12$ & - & Ludlow e Wilkins (2016) \\
\hline & $\%$ & $92 \% *$ & & & $50 \% *$ & & \\
\hline & $\chi^{2}$ & 17,71 & & & 9,05 & & \\
\hline & $\mathrm{p}$ & 0,0001 & & & 0,003 & & \\
\hline \multirow{4}{*}{ 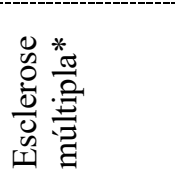 } & $\mathrm{n} / \mathrm{N}$ & $20 / 26$ & - & - & - & $8 / 26$ & Wright et al. (2007) \\
\hline & $\%$ & $77 \% *$ & & & & $31 \% *$ & \\
\hline & $\chi^{2}$ & 20,83 & & & & 31,16 & \\
\hline & $\mathrm{p}$ & 0,0001 & & & & 0,0001 & \\
\hline
\end{tabular}

*Chi-Quadrado com diferença significativa em relação ao grupo de Crianças e Adolescentes, $\mathrm{p}<0,05$.

${ }^{\Delta}$ Limitação: $54 \%$ de sujeitos com problemas refrativos não corrigidos e RRT com fonte ampliada. 
Tabela 4.

Síntese da Meta-análise da prevalência do ganho na velocidade de leitura no RRT com o uso da lâmina espectral ideal

\begin{tabular}{|c|c|c|c|c|c|c|}
\hline \multirow{2}{*}{\multicolumn{2}{|c|}{ População }} & \multicolumn{5}{|c|}{ Melhora na velocidade de leitura no teste RRT } \\
\hline & & $\geq 5 \%$ & $\geq 8 \%$ & $\geq 10 \%$ & $\geq 15 \%$ & $\geq 25 \%$ \\
\hline \multirow{2}{*}{$\begin{array}{c}\text { Amostra } \\
\text { populacional }\end{array}$} & $\begin{array}{c}\text { Crianças e } \\
\text { Adolescentes }\end{array}$ & $33 \%$ & $23 \%$ & $18 \%$ & $13 \%$ & $5 \%$ \\
\hline & Adultos & $34 \%$ & $46 \% *$ & $19 \%$ & - & $2 \% *$ \\
\hline \multirow{2}{*}{$\begin{array}{l}\text { Dificuldade } \\
\text { de leitura* }\end{array}$} & Crianças* & $41 \%$ & $36 \% *$ & $30 \% *$ & $17 \%$ & $10 \% *$ \\
\hline & Adultos* & $57 \% *$ & $63 \% *$ & $44 \% *$ & - & - \\
\hline \multirow{3}{*}{ Transtornos* } & Autista* & $74 \% *$ & $56 \% *$ & $50 \% *$ & $25 \%$ & $30 \% *$ \\
\hline & Tourette* & $92 \% *$ & & & $50 \% *$ & \\
\hline & Esclerose múltipla* & $77 \% *$ & & & & $31 \% *$ \\
\hline
\end{tabular}

\section{Discussão}

\section{Prevalência por meio do autorrelato}

De acordo com onze amostras de sete estudos $(N=1756), 63 \%$ das crianças avaliaram que houve melhora na qualidade e/ou conforto visual com o uso das lâminas espectrais. Ou seja, seis a cada dez crianças preferiram ler com a lâmina. Todos os sete estudos populacionais confrontaram previamente o participante com estímulos visuais estressores para eliciar respostas mais fidedignas e melhorar a autoconsciência das distorções visuais na leitura.

Há um elevado e significativo índice de universitários (89\%) que relatam melhora visual com o uso das lâminas. Evans e Joseph (2002) verificaram que 89\% dos universitários relatam melhora qualitativa da leitura com as lâminas, porém a amostra foi espontânea e não representativa da população, uma vez que estudantes com suspeitas de dificuldade visuoperceptual na leitura tendem a se voluntariar em pesquisas. No entanto, essa prevalência é similar aos 88\% encontrados por Miller (1985), o que sugere que Universitários, por possuírem alta demanda de leitura, valorizam mais o conforto visual provido pelas lâminas espectrais.

Significativamente mais crianças com dificuldade de leitura $(87 \%)$ relatam melhora visual com as lâminas espectrais do que o grupo de comparação (63\%). Dentre 
todas as referidas estimativas, há uma provável proporção de falso positivos, já que o autorrelato de melhora é um critério qualitativo, podendo o participante selecionar a lâmina por razões estéticas (escolha da cor favorita) ou motivacionais (pela novidade em si ou pelo efeito placebo inerente a qualquer método), apesar de estudos terem demonstrado que essas variáveis possuem pouca influência (Bouldoukian et al., 2002; A. K. Ludlow, A. Wilkins, \& P. Heaton, 2008; Wilkins et al., 2001). Importante destacar que normalmente em pesquisas, especialmente com adultos, há uma maior tendência dos participantes se voluntariarem devido a suspeitas prévias de algum transtorno, o que aumenta a incidência em comparação a uma amostra representativa e aleatória da população geral.

Muitos leitores selecionarão uma lâmina espectral devido a maior qualidade visual e contraste do texto, no entanto não a utilizarão a longo prazo, pois não apresentam distorções visuais que necessitam ser remediadas. Assim, 63\% das crianças e $89 \%$ dos adultos apresentam melhora do conforto visual leitor com a lâmina espectral. No entanto, esses leitores não necessariamente utilizarão a lâmina no cotidiano de sala de aula ou apresentarão um ganho no desempenho de leitura, critérios importantes para o diagnóstico de um distúrbio do processamento visual subjacente.

\section{Prevalência por meio do uso prolongado}

O uso prolongado das lâminas espectrais se refere ao seu uso voluntário e continuado a longo prazo, sendo um bom indicador da prevalência populacional de seu efeito terapêutico. Esse dado evidencia que o leitor a utiliza espontaneamente no dia a dia, a considerando importante para melhorar a qualidade visual de sua leitura, o que reduz a proporção de falso positivo encontrado na prevalência por meio do autorrelato. Deve-se levar em consideração que algumas pessoas, mesmo sendo beneficiadas pela lâmina espectral, descontinuam o seu uso por a considerar constrangedora, escura ou inconveniente (Williams, Lecluyse, \& Rock-Faucheux, 1992).

Em média, a prevalência reduziu de $63 \%$ para $29 \%$ após $2-4$ meses de uso das lâminas (Jeanes et al., 1997; Scott et al., 2002; Wilkins et al., 1996), e de 58\% para 31\% após 8-10 meses (Jeanes et al., 1997; Wilkins et al., 2001). Ou seja, 29,5\% (n/ $\mathrm{N}=$ 299/1014) das crianças em idade escolar utilizaram espontaneamente as lâminas como ferramenta educacional no cotidiano de sala de aula. Esse índice é um dos mais 
importantes aqui descritos, pois provê validade prática para uso das lâminas espectrais, evidenciando que grande proporção de pessoas continua as usando mesmo após o declínio do fator motivacional.

\section{Prevalência por meio do ganho no desempenho de leitura pelo RRT}

O desempenho de leitura é um importante parâmetro para verificar a intensidade dos benefícios das lâminas espectrais e a probabilidade do seu uso continuado. Qualificar a velocidade de leitura, por meio do RRT, é uma forma objetiva de analisar a eficiência e a prevalência do ganho visual leitor com o uso das lâminas, o que tornou esse teste o mais utilizado nos estudos científicos na área. O RRT foi criado após Tyrrell et al. (1995) terem percebido diferença significativa nos sintomas visuais e na leitura somente após 10 minutos, sendo fundamental criar um teste que possua reduzido caráter linguístico, focado nos processamentos visuais necessários para a leitura, e que consiga detectar diferença em um menor tempo de aplicação.

Para quantificar o efeito das lâminas espectrais na velocidade de leitura de crianças brasileiras, o teste RRT foi recentemente utilizado em duas dissertações de mestrados, realizadas na Pontifícia Universidade Católica de São Paulo (Garcia, 2016) e na Universidade Federal de Alfenas (Leão, 2018). Garcia (2016) investigou uma amostra de 68 crianças no $5^{\circ}$ e $6^{\circ}$ ano do Ensino Fundamental, cujos resultados foram publicados por Garcia et al. (2017). A dissertação de Leão (2018) investigou uma amostra de 58 crianças do $3^{\circ}$ e $4^{\circ}$ ano com dificuldade de leitura. Em ambas as dissertações foram excluídas da amostra crianças com problemas refrativos não corrigidos. Problemas refracionais afetam a acuidade visual, impactando na acurácia e fluência leitora, o que limita a análise do ganho real na velocidade de leitura. Esse rigor na seleção da amostra não foi adotado por Ritchie et al. (2011), onde 54,3\% das 61 crianças escocesas com dificuldade de leitura apresentavam problemas ópticos não corrigidos. Ademais, a metodologia utilizou o teste RRT com fonte ampliada, anulando o fator estressor induzido pelo teste padrão, que estipula a impressão com letra pequena e espaçamento simples, preconizado por Wilkins et al. (1996).

O uso da lâmina espectral ideal melhorou a velocidade de leitura para $34 \%$ (n/N $=338 / 1006)$ da população geral, com $18 \%(\mathrm{n} / \mathrm{N}=57 / 317)$ demonstrando ganhos moderados. O critério de ganho de pelo menos $15 \%$ no RRT é considerado como uma 
melhora além da variabilidade intra-individual (Garcia et al., 2017), porém apenas quatro de treze estudos explicitaram a frequência da amostra com esse ganho na taxa de leitura. É possível que o critério mais rigoroso de ganho na velocidade de leitura (mínimo de $25 \%$ de aumento) não tenha refletido a real proporção da população com forte melhora na leitura, especialmente na população de adultos, devido ao efeito de teto do RRT, já que há um limite fonoarticulatórios para uma leitura em voz alta.

Estudos verificaram que quanto maior a dificuldade, desconforto e presença de distorções, maior é o ganho na velocidade de leitura com o uso das lâminas espectrais (Garcia et al., 2017; Hollis \& Allen, 2006; Tyrrell et al., 1995; Wilkins et al., 2001). A dificuldade de leitura é um dos maiores indicadores tanto para o autorrelato de melhora na qualidade de acesso visual ao texto quanto para o ganho na velocidade de leitura com o uso das lâminas (Kriss \& Evans, 2005; Singleton \& Henderson, 2007b; Tyrrell et al., 1995). A presente meta-análise verificou que $87 \%$ dos sujeitos com dificuldade de leitura optaram pelo uso de lâminas espectrais, tendo $30 \%$ das crianças e $44 \%$ dos adultos melhorado a velocidade de leitura em pelo menos $10 \%$, valores que são significativamente maiores do que os observados nos respectivos grupos controle.

Transtornos que provocam hiperexcitação cortical tendem a ser beneficiados no desempenho de leitura com o uso das lâminas, cujo efeito seria uma atenuação da sobrecarga sensorial visual, frequente no Transtorno do Espectro Autista e esclerose múltipla. A alta proporção de pacientes com o Transtorno do Espectro Autista (74\%) com ganhos de 5\% no RRT se deve à maior sensibilidade a estímulos sensoriais (olfativo, auditivo, gustativo, tátil e visual) que se manifesta e impacta desfavoravelmente na vida cotidiana desses pacientes (Al-Heizan, AlAbdulwahab, Kachanathu, \& Natho, 2015; Cermak, Curtin, \& Bandini, 2010; Leekam, Nieto, Libby, Wing, \& Gould, 2007).

Com o uso das lâminas espectrais, pacientes com esclerose múltipla apresentam melhora significativa, no curto e em longo prazo, na leitura e na procura visual, com redução dos sintomas de estresse visual (Newman Wright et al., 2007). Esses autores atribuem parte desse efeito a transtornos que provocariam hiperexcitabilidade cortical, como a alta frequência de enxaqueca (comorbidade de $56 \%$ ) ou vivência de pelo menos uma crise epilética (4\%) e não pela esclerose múltipla em si.

Importante destacar que a análise da velocidade de leitura deve ser complementada com outros dados clínico e quantitativos, como por exemplo os de oculomotricidade, uma vez que a sensibilidade do RRT em predizer o uso prolongado das 
lâminas varia de 60 a 73\% (Northway, 2003; Wilkins et al., 1996; Wilkins et al., 2001). Ademais, mesmo o RRT sendo um bom indicador do uso prolongado, muitos pacientes poderiam não apresentar ganhos na velocidade de leitura, mas somente no conforto visual (Northway, 2003).

A fluência de leitura é composta por três componentes primários: (a) acurácia/precisão na decodificação, (b) velocidade/automaticidade no reconhecimento de palavras e (c) o uso apropriado de recursos prosódicos (Kuhn \& Stahl, 2003). Embora o benefício do uso de overlays pareça proporcionalmente pequeno, o RRT avalia apenas o componente de velocidade de leitura, que é um dos três componentes da fluência de leitura, junto com a acurácia e a prosódia. Essa restrição técnica restringe o RRT aos aspectos visuais da leitura, reduzindo os aspectos linguísticos, uma vez que as palavras são familiares (alta frequência de ocorrência) e com curto comprimento ( 2 a 4 letras). As lâminas espectrais beneficiam outros aspectos envolvidos na leitura, como o contraste do texto impresso no papel (figura/fundo), atenuação das diversas distorções que se manifestam progressivamente e no conforto visual pela redução da percepção de brilho e do esforço visual. Estas contribuições não contempladas pelo RRT.

A partir dos resultados no RRT, é possível depreender que os benefícios proporcionados pelas lâminas espectrais se apresentam dentro de uma escala de intensidade. Essa classificação se adequa à tendência corrente que considera o conceito de dimensionalidade na descrição das disfunções do desenvolvimento (ex., Transtorno do Espectro Autista). Neste contexto, a descrição dos benefícios aferidos pelos usuários de overlays passa a ser feita dentro de um contínuo que varia entre ganhos leves $(5 \%)$, moderados $(10 \%)$ ou robustos $(25 \%)$, auxiliando na compreensão da ampla variação dos sintomas visuoperceptuais de pessoa para pessoa.

\section{Considerações finais}

A lâmina espectral se mostrou um instrumento de intervenção efetiva e de fácil acesso para melhorar a qualidade de leitura de crianças e adultos. Na presente metaanálise, foi verificado que seis em cada dez crianças (63\%) e nove em cada dez adultos (89\%) relataram melhora na qualidade e/ou conforto visual com o uso das lâminas espectrais. Caso a lâmina fosse fornecida, $30 \%$ da população faria o seu uso prolongado durante tarefas diárias de leitura. Sob uso de lâminas, 33\% da população melhorou a 
velocidade de leitura, quantificada pelo RRT, tendo 18\% apresentado ganhos de moderados a robustos, impactando na dinâmica do aprendizado escolar. Esse ganho no desempenho leitor não pode ser explicado por problemas ópticos, por razões estéticas ou motivacionais. Os transtornos que mais apresentaram ganhos foram o Transtorno do Espectro Autista, Síndrome de Tourette, Esclerose Múltipla e Dificuldade de Leitura.

\section{Referências}

Al-Heizan, M. O., AlAbdulwahab, S. S., Kachanathu, S. J., \& Natho, M. (2015). Sensory processing dysfunction among Saudi children with and without autism. $J$ Phys Ther Sci, 27(5), 1313-1316. doi: 10.1589/jpts.27.1313

Allen, P. M., Gilchrist, J. M., \& Hollis, J. (2008). Use of visual search in the assessment of pattern-related visual stress (PRVS) and its alleviation by colored filters. Invest Ophthalmol Vis Sci, 49(9), 4210-4218. doi: 10.1167/iovs.07-1587

Bernal, M. (2011). Validación del método Irlen en escolares de 6 a 8 años, con trastornos de lectura. Universidad de Cuenca, Cuenca. Retrieved from http://dspace.ucuenca.edu.ec/handle/123456789/2213

Bouldoukian, J., Wilkins, A. J., \& Evans, B. J. (2002). Randomised controlled trial of the effect of coloured overlays on the rate of reading of people with specific learning difficulties. Ophthalmic Physiol Opt, 22(1), 55-60.

Cermak, S. A., Curtin, C., \& Bandini, L. G. (2010). Food selectivity and sensory sensitivity in children with autism spectrum disorders. J Am Diet Assoc, 110(2), 238246. doi: 10.1016/j.jada.2009.10.032

Chase, C., Ashourzadeh, A., Kelly, C., Monfette, S., \& Kinsey, K. (2003). Can the magnocellular pathway read? Evidence from studies of color. Vision Res, 43(10), 1211-1222. doi: http://dx.doi.org/10.1016/S0042-6989(03)00085-3

Croyle, L. (1998). Rate of reading, visual processing, colour and contrast. Aust J Learn Diffic, 3(3), 13-21. doi: 10.1080/19404159809546566

Evans, B. J., \& Joseph, F. (2002). The effect of coloured filters on the rate of reading in an adult student population. Ophthalmic Physiol Opt, 22(6), 535-545. 
Evans, B. J., Wilkins, A. J., Brown, J. A., Busby, A., Wingfield, A., Jeanes, R., \& Bald, J. (1996). A preliminary investigation into the aetiology of Meares-Irlen syndrome. Ophthalmic Physiol Opt, 16(4), 286-296.

Garcia, A. C. O. (2016). Efeito das Lâminas Espectrais sobre Desempenho da Leitura em Escolares do Ensino Fundamental,Ano de Obtenção: 2016. (Mestrado), Pontifícia Universidade Católica de São Paulo, PUC/SP, Brasil.

Garcia, A. C. O., Momensohn-Santos, T. M., \& Vilhena, D. A. (2017). Effects of Spectral Overlays on Reading Performance of Brazilian Elementary School Children. Folia Phoniatrica et Logopaedica, 69(5-6), 219-225.

Hlengwa, N., Moonsamy, P., Ngwane, F., Nirghin, U., \& Singh, S. (2017). The effect of color overlays on the reading ability of dyslexic children. Indian J Ophthalmol, 65(8), 772-773. doi: 10.4103/ijo.IJO_541_16

Hollingsworth, R. S., Ludlow, A. K., Wilkins, A. J., Calver, R. I., \& Allen, P. M. (2015). Visual performance and the use of colored filters in children who are deaf. Optom Vis Sci, 92(6), 690-699. doi: 10.1097/OPX.0000000000000595

Hollis, J., \& Allen, P. M. (2006). Screening for Meares-Irlen sensitivity in adults: can assessment methods predict changes in reading speed? Ophthalmic Physiol Opt, 26(6), 566-571. doi: 10.1111/j.1475-1313.2006.00401.x

Irlen, H., \& Lass, M. J. (1989). Improving reading problems due to symptoms of Scotopic Sensitivity Syndrome using Irlen lenses and overlays. Education, 109(4), 413-417.

Jeanes, R., Busby, A., Martin, J., Lewis, E., Stevenson, N., Pointon, D., \& Wilkins, A. J. (1997). Prolonged use of coloured overlays for classroom reading. Br J Psychol, 88 ( Pt 4), 531-548.

Kriss, I., \& Evans, B. J. (2005). The relationship between dyslexia and Meares-Irlen Syndrome. J Res Read, 28(3), 350-364. doi: 10.1111/j.1467-9817.2005.00274.x

Kuhn, M., \& Stahl, S. (2003). Fluency: A review of developmental and remedial practices. Journal of educational psychology, 95(1), 3-21.

Leão, D. M. P. (2018). Uso de Lâminas Espectrais por Alunos do Ensino Fundamental com Síndrome de Irlen no Município de Alfenas-MG. (Mestrado), Universidade Federal de Alfenas, UNIFAL-MG, Brasil.

Leekam, S. R., Nieto, C., Libby, S. J., Wing, L., \& Gould, J. (2007). Describing the sensory abnormalities of children and adults with autism. J Autism Dev Disord, 37(5), 894-910. doi: 10.1007/s10803-006-0218-7 
Ludlow, A. K., Taylor-Whiffen, E., \& Wilkins, A. J. (2012). Coloured filters enhance the visual perception of social cues in children with autism spectrum disorders. ISRN Neurol, 2012, 298098. doi: 10.5402/2012/298098

Ludlow, A. K., Wilkins, A., \& Heaton, P. (2008). Colored overlays enhance visual perceptual performance in children with autism spectrum disorders. Research in Autism Spectrum Disorders, 2(3), 498-515.

Ludlow, A. K., Wilkins, A. J., \& Heaton, P. (2006). The effect of coloured overlays on reading ability in children with autism. J Autism Dev Disord, 36(4), 507-516. doi: 10.1007/s10803-006-0090-5

Ludlow, A. K., Wilkins, A. J., \& Heaton, P. (2008). Colored overlays enhance visual perceptual performance in children with autism spectrum disorders. Research in Autism Spectrum Disorders, 2(3), 498-515.

Monger, L., Wilkins, A. J., \& Allen, P. M. (2015). Identifying visual stress during a routine eye examination. Journal of Optometry, 8(2), 140-145. doi: http://dx.doi.org/10.1016/j.optom.2014.10.001

Newman Wright, B., Wilkins, A., \& Zoukos, Y. (2007). Spectral filters can improve reading and visual search in patients with multiple sclerosis. $J$ Neurol, 254(12), 17291735. doi: 10.1007/s00415-007-0648-y

Nichols, S. A., McLeod, J. S., Holder, R. L., \& McLeod, H. S. (2009). Screening for dyslexia, dyspraxia and Meares-Irlen syndrome in higher education. Dyslexia, 15(1), 42-60. doi: 10.1002/dys.382

Northway, N. (2003). Predicting the continued use of overlays in school children--a comparison of the Developmental Eye Movement test and the Rate of Reading test. Ophthalmic Physiol Opt, 23(5), 457-464.

Ritchie, S. J., Della Sala, S., \& McIntosh, R. D. (2011). Irlen colored overlays do not alleviate reading difficulties. Pediatrics, 128(4), e932-938. doi: 10.1542/peds.20110314

Scott, L., McWhinnie, H., Taylor, L., Stevenson, N., Irons, P., Lewis, E., . . Wilkins, A. J. (2002). Coloured overlays in schools: orthoptic and optometric findings. Ophthalmic Physiol Opt, 22(2), 156-165.

Singleton, C., \& Henderson, L.-M. (2007a). Computerised screening for visual stress in reading. $J$ Res Read, 30(3), 316-331. doi: 10.1111/j.1467-9817.2007.00341.x 
Singleton, C., \& Henderson, L.-M. (2007b). Computerized screening for visual stress in children with dyslexia. Dyslexia, 13(2), 130-151. doi: 10.1002/dys.329

Solan, H. A., Ficarra, A., Brannan, J. R., \& Rucker, F. (1998). Eye movement efficiency in normal and reading disabled elementary school children: effects of varying luminance and wavelength. J Am Optom Assoc, 69(7), 455-464.

Stein, J., \& Walsh, V. (1997). To see but not to read; the magnocellular theory of dyslexia. Trends Neurosci, 20(4), 147-152.

Tyrrell, R., Holland, K., Dennis, D., \& Wilkins, A. J. (1995). Coloured overlays, visual discomfort, visual search and classroom reading. Journal of Research in Reading, 18(1), 10-23. doi: 10.1111/j.1467-9817.1995.tb00064.x

Whichard, J. A., Feller, R. W., \& Kastner, R. (2000). The Incidence of Scotopic Sensitivity Syndrome In Colorado Inmates. Journal of Correctional Education, 51(3), 294-299.

Whitaker, L., Jones, C. R. G., Wilkins, A. J., \& Roberson, D. (2016). Judging the Intensity of Emotional Expression in Faces: the Effects of Colored Tints on Individuals With Autism Spectrum Disorder. Autism Research, 9(4), 450-459. doi: doi:10.1002/aur.1506

Wilkins, A. J. (1994). Overlays for classroom and optometric use. Ophthalmic Physiol Opt, 14(1), 97-99.

Wilkins, A. J. (1995). Visual stress. Oxford ; New York: Oxford University Press.

Wilkins, A. J., Jeanes, R. J., Pumfrey, P. D., \& Laskier, M. (1996). Rate of Reading Test: its reliability, and its validity in the assessment of the effects of coloured overlays. Ophthalmic Physiol Opt, 16(6), 491-497. doi: 10.1046/j.1475-1313.1996.96000282.x

Wilkins, A. J., \& Lewis, E. (1999). Coloured overlays, text, and texture. Perception, 28(5), 641-650.

Wilkins, A. J., Lewis, E., Smith, F., Rowland, E., \& Tweedie, W. (2001). Coloured overlays and their benefit for reading. J Res Read, 24(1), 41-64. doi: 10.1111/14679817.00132

Williams, M. C., Lecluyse, K., \& Rock-Faucheux, A. (1992). Effective interventions for reading disability. J Am Optom Assoc, 63(6), 411-417. 